\title{
Fractal scattering in a radiation field
}

\author{
Yi-Der Lin, Alex Barr, Kyungsun Na, and Linda E. Reichl \\ Center for Complex Quantum Systems and Department of Physics, University of Texas at Austin, Austin, Texas 78712, USA
}

(Received 1 February 2011; published 31 May 2011)

\begin{abstract}
We examine the transfer matrix and the stepping times for chaotic scattering of the excess electron in the chlorine ion $\mathrm{Cl}^{-}$from the chlorine atom. We use this information to determine the fraction of incident trajectories that are reflected and transmitted for those electrons caught in the tendrils of the chaotic scattering process.
\end{abstract}

DOI: 10.1103/PhysRevE.83.056217

PACS number(s): 05.45.-a, 32.90.+a

\section{INTRODUCTION}

Chaos can profoundly affect the scattering dynamics of classical systems, and it can exert significant influence on the quantum analog of the scattering dynamics. A monochromatic radiation field coupled to the nonlinear dynamics of an otherwise field-free scattering process gives rise to an extremely rich scattering dynamics governed by a fractal set of singularities that is embedded in all dynamical functions involved with the scattering process. This fractal set of singularities occurs when the incoming particle asymptotes intersect the tangles of the invariant manifolds in the asymptotic region. The recipe for extracting information about chaotic scattering processes and the underlying fractal structure that governs that dynamics has been developed by Jung and others for a variety of model systems [1-5]. The method described here for obtaining the underlying fractal structure for chaotic scattering process can also be applied to scattering processes in the absence of a radiation field, as has been shown in Ref. [6], where the chaotic scattering dynamics of a molecular system is analyzed. More general reviews of scattering chaos can be found in Refs. [7,8].

A particularly fruitful system for studying fractal scattering dynamics, both classically [3-5] and quantum mechanically $[9,10]$, is the negative chlorine ion $\mathrm{Cl}^{-}$in the presence of a radiation field. In Refs. [3-5] the fractal structure of the scattering dynamics for this system was studied in great detail. However, one important physical aspect was not addressed, namely, how to determine the fraction of trajectories that is transmitted through the scattering region versus the fraction that is reflected from the scattering region. That is the issue we address in this paper.

We begin in Sec. II with a discussion of properties of the Hamiltonian that we use to study the fractal scattering process. In Sec. III we define a "fundamental region" using invariant manifolds of the driven potential, and we analyze the fractal network of tendrils formed by these invariant manifolds. In Sec. IV we describe the symbolic dynamics that is used to develop a branching tree that reflects the complexity of the network of tendriles in the asymptotic region of the phase space. We also define a transfer matrix for the branching tree and use it to give an estimate of the fraction of orbits that are reflected and transmitted for a given incident direction. Finally, in Sec. V we make some concluding remarks.

\section{THE HAMILTONIAN}

The model system we consider is that of an electron of mass $m$ and charge $q$, in the presence of a monochromatic radiation field whose electric field $E$ is linearly polarized along the direction of motion of the electron. The electron, while being driven by the radiation, scatters from a one-dimensional inverted Gaussian atomic potential. The Hamiltonian can be written

$$
H_{1}\left(p^{\prime}, x^{\prime}, t\right)=\frac{\left(p^{\prime}\right)^{2}}{2 m}-V_{0} e^{-\left(x^{\prime} / \delta\right)^{2}}-x^{\prime} F \sin (\omega t),
$$

where $p^{\prime}$ and $x^{\prime}$ are the momentum and displacement of the electron in the laboratory frame and $F=q E$. We can transform from the laboratory frame $\left\{p^{\prime}, x^{\prime}\right\}$ to a reference frame $\{p, x\}$ moving with the electron [the Kramers-Henneberger frame $(\mathrm{KH})[11,12]]$ via a canonical transformation whose generating function is $\mathcal{F}_{3}\left(p^{\prime}, x, t\right)=-p^{\prime} x+\frac{F}{\omega}[-x \cos (\omega t)+$ $\left.\frac{p^{\prime}}{m \omega} \sin (\omega t)\right]$ [13]. Then $p=-\frac{\partial \mathcal{F}_{3}}{\partial x}$ and $x^{\prime}=-\frac{\partial \mathcal{F}_{3}}{\partial p^{\prime}}$, and the transformed Hamiltonian $H_{2}$ is given by $H_{2}=H_{1}+\frac{\partial \mathcal{F}_{3}}{\partial t}$. We can further transform this time-periodic Hamiltonian to a time-independent Hamiltonian with coordinates $\{p, x, J, \phi\}$ by letting $\phi=\omega t$ and writing

$$
H_{3}(p, x, J, \phi)=\frac{p^{2}}{2 m}+V_{0} e^{-(x-\alpha(\phi) / \delta)^{2}}+G(\phi)+\omega J,
$$

where $\quad \alpha(\phi)=\alpha_{0} \sin (\phi), \quad \alpha_{0}=F / \omega^{2}, \quad$ and $\quad G(\phi)=$ $\frac{F^{2}}{2 m \omega^{2}}\left[2 \sin (\phi)^{2}-\cos (\phi)^{2}\right]$. The action variable $J$ for the radiation field evolves independently of the motion of the electron, and $\dot{\phi}=\omega$ at all times. In the $\mathrm{KH}$ frame, the inverted Gaussian potential oscillates back and forth, and, in the asymptotic region, the electron motion is free motion since $\dot{p}=0$ and $\dot{x}=p$. The Hamiltonian is invariant under the generalized symmetry transformation $x \rightarrow-x$ and $\phi \rightarrow \phi+\pi$, but not under the transformation $x \rightarrow-x$ alone. Therefore, there is an asymmetry in the scattering dynamics of this system. As we will see below, the fraction of incident particles that are transmitted and reflected will differ depending on the incident direction.

In subsequent sections, we use atomic units so the electron mass $m=1$ a.u., and we choose $V_{0}=0.27035$ a.u., $\delta=$ 2 a.u., $\omega=0.65$ a.u., and $\alpha_{0}=0.9$ a.u., which have been shown to describe the quantum behavior of a negative chlorine ion $\mathrm{Cl}^{-}$in the presence of a laser field [3,14-16].

\section{FRACTAL SCATTERING}

The fractal structure of the scattering process can be seen in a Poincaré surface of section (PSS) of the dynamics. We solve Hamilton's equations in the KH frame and plot $p$ and $x$ each time $\phi=\pi / 2+n 2 \pi$, where integer $n=0,1,2, \ldots$ The 

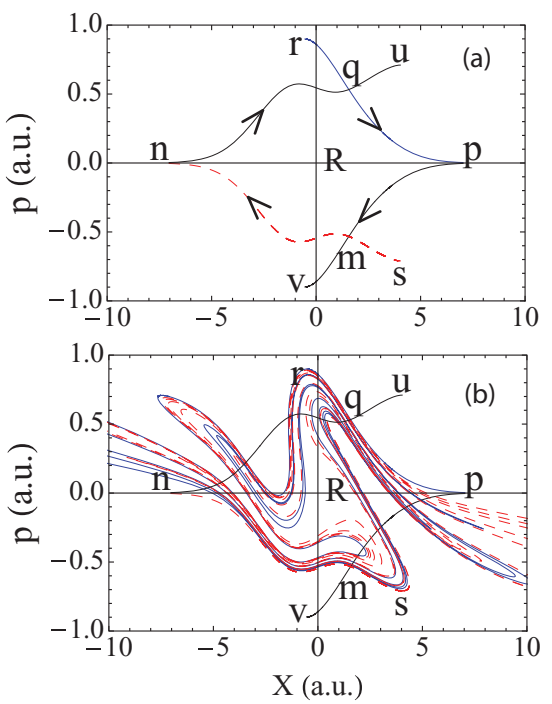

FIG. 1. (Color online) A Poincaré surface of section with $p$ and $x$ plotted each time $\phi=\pi / 2$. (a) The curves n-u and s-n (r-p and $\mathrm{p}-\mathrm{v})$ are segments of the unstable and stable manifolds of $F_{L}\left(F_{R}\right)$, respectively. The fundamental region is formed by the curves n-q, q-p, n-m, and m-p. (b) The segments of the stable manifolds $r-p$ (blue-solid line) and n-s (red dashed line) are integrated backward in time for four iterations of the PSS.

inverted Gaussian system, in the absence of radiation, has three primary period $-2 \pi$ periodic orbits (fixed points of the PSS): a stable periodic orbit at $(p=0, x=0)$, an unstable periodic orbit $\left(F_{L}\right)$ at $(p=0, x=-\infty)$, and an unstable periodic orbit $\left(F_{R}\right)$ at $(p=0, x=+\infty)$. We define a fundamental region $R$, in the PSS, whose boundaries are given by segments of the invariant manifolds of the outer fixed points of the system. The invariant manifolds and the fundamental area $R$ are shown in Fig. 1(a). The invariant manifolds are located by taking a line of initial points with fixed $x$ but varying $p>0$ near $p=0$ and in the neighborhood of the separatrix in the absence of radiation. We evolve the points in time and determine which points escape and which points do not escape. The point separating these two behaviors is on the invariant manifold. This process is repeated for different values of $x$ until a segment of the manifold is mapped out. The curves n-u and s-n are segments of the unstable and stable manifolds of $F_{L}$, respectively. The curves $\mathrm{r}-\mathrm{p}$ and $\mathrm{p}-\mathrm{v}$ are segments of the stable and unstable manifolds of $F_{R}$, respectively. The fundamental area is defined by the curves $n-q, q-p, n-m$, and $m-p$. Because the invariant manifolds approach the $p=0$ axis exponentially as $x \rightarrow \pm \infty$, it is sufficient to consider the segments shown in the figure.

In Fig. 1(b) we show four iterations backward in time of the segments $r-p$ and s-n of the stable manifolds. The stable manifolds cannot intersect themselves, and they cannot intersect each other. However, they can intersect the unstable manifolds. The curves, as they are iterated backward in time, conserve area. These constraints cause them to form a fractal network of tendrils as $t \rightarrow-\infty$.

We can distinguish points associated with the asymptotic region from those associated with the fundamental region. This becomes clear in Figs. 2(a) and 2(b), where the shaded regions with positive indices contain points that lie outside
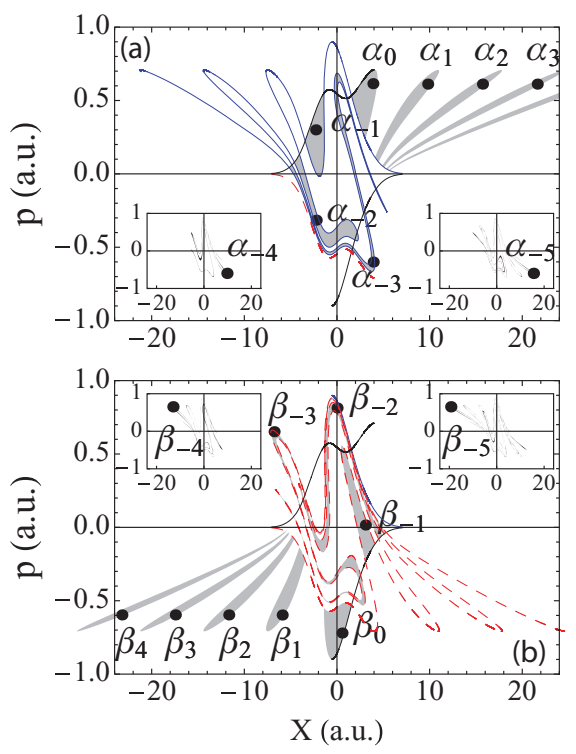

FIG. 2. (Color online) (a) The points labeled $\alpha_{-5}, \ldots, \alpha_{+3}$ show the movement of a single point $\alpha_{n}$ incident from the right for nine iterations of the PSS. Initially, the point lies in a tangle in the asymptotic region indicated by the shaded region. The point $\alpha$ takes two steps inside the fundamental area $R$ as the PSS is iterated forward in time, and eventually the entire shaded region is mapped back to the right, and all points in it are reflected. (b) The points labeled $\beta_{-5}, \ldots, \beta_{+4}$ show the movement of a single point, $\beta_{n}$, incident from the left for ten iterations of the PSS. The point $\beta$ takes one step inside the fundamental region before being reflected back to the left.

the fundamental region. Iterations of these shaded regions are shown for times ranging from $t=-5$ to +4 (nine iterations of the PSS). As the map is iterated backward in time, the shaded regions form more and more complex filaments that cut across the fundamental region. As the shaded regions are iterated forward in time, they approach their respective asymptotic regions. In Fig. 2(a) we follow the point $\alpha_{n}(-5 \leqslant n \leqslant 4)$, which is incident from the right at time $n=-5$, but happens initially to be inside one of the tendrils in the asymptotic region at that time. In Fig. 2(b) we follow the point $\beta_{n}(-5 \leqslant n \leqslant 4)$, which is incident from the left at time $n=-5$ and is initially inside a tendril in the asymptotic region at that time.

Careful inspection of Fig. 1(b) and Figs. 2(a) and 2(b) shows that the "backward in time" filaments from the two stable manifolds become interlaced in the tendrils that extend backward in time to the upper left, and backward in time to the lower right. A particle, incident from the left, that initially lies in one of these "backward in time" tendrils can either be transmitted through the scattering region toward $x=+\infty$ or it can be reflected from the scattering region toward $x=-\infty$, depending whether it initially lies in a filament of the type shown in Fig. 2(a) or that shown in Fig. 2(b).

In Fig. 3(a) we show the evolution in time of a line of points initially lying between $x=-10.5$ and -10.1 for a particle incident from the left. The line of initial conditions, in the distant past, was chosen to lie across a tendril far out in the asymptotic region to the left. Figure 3(a) shows the line of initial conditions as it is evolved forward in time, for four 

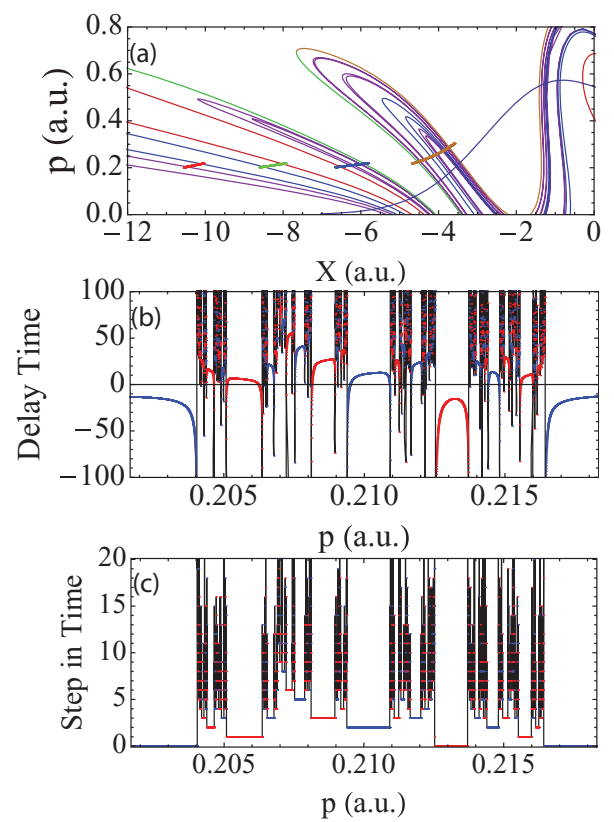

FIG. 3. (Color online) (a) The evolution in time of a line of initial conditions for a particle incident from the left. The plot shows four iterations of the PSS. One more iteration would show the line of points stepping inside the fundamental region $R$. (b) The delay time [the time each point is delayed before being transmitted (red) or reflected (blue)] for the line of initial points shown in (a). (c) A plot of number of steps taken inside the fundamental region before being transmitted (red) or reflected (blue), for those trajectories that take fewer than 20 steps inside $R$. A complete plot would show step numbers ranging from one to infinity and a fractal mixed structure of transmitted and reflected points.

iterations of the PSS, and approaches the fundamental region $R$. In the next iteration the line of points will step into the fundamental region. In Fig. 3(b) we show the time it takes various points in this line of initial conditions to leave the fundamental region (the delay time) regardless of whether the points are transmitted or reflected. The delay time forms a fractal set characteristic of this scattering process. In Fig. 3(c) we show a plot of the number of steps taken inside $R$, by the points initially lying between $x=-10.5$ and -10.1 in Fig. 3(a). We show only results for points that take fewer than 20 steps inside $R$. The step numbers for all initial points take values ranging from zero to infinity and form a fractal that is an image of the fractal structure of the scattering region.

\section{SYMBOLIC DYNAMICS}

A symbolic dynamics for this scattering process can be constructed by examining the structure of the gaps that are cut into the unstable manifolds $n-q$ and $p-m$ by the tendrils formed by the stable manifolds $r-p$ and s-n as they are iterated backward in time. In Fig. 4(a) we show the first iteration of the stable manifolds backward in time. The shaded regions show basins of the asymptotic regions that cut through the fundamental region $R$ on this first iteration of the PSS. The shaded regions cut two segments out of the unstable manifold of $F_{L}$ and cut one segment out of the unstable manifold of $F_{R}$. We label the three remaining pieces of the unstable manifold
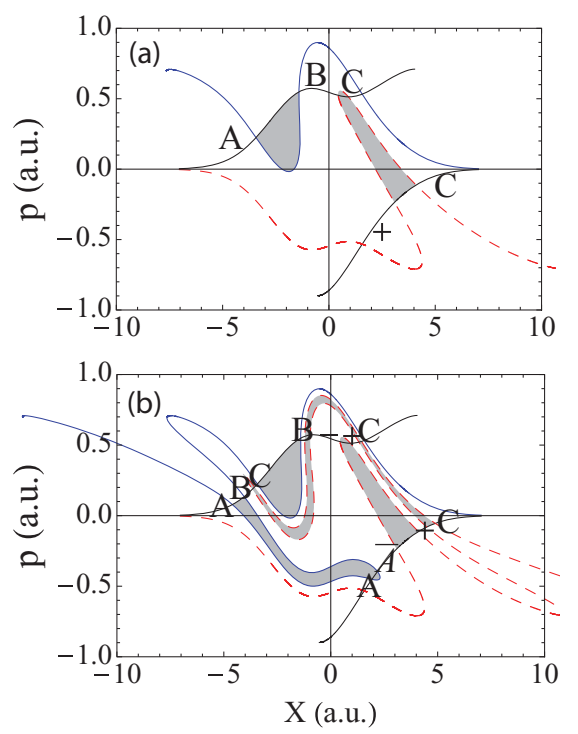

FIG. 4. (Color online) (a) The first iteration of the stable manifolds backward in time. The shaded regions are basins of the asymptotic regions that cut through the fundamental region $R$ on the first iteration of the PSS. The shaded regions cut two segments out of the unstable manifold of $F_{L}$ and cut one segment out of the unstable manifold of $F_{R}$. The three remaining pieces of the unstable manifold of $F_{L}$ are labeled "A," "B," and "C." The two remaining pieces of the unstable manifold of $F_{R}$ are labeled "+" and "C." (b) The second iteration of the stable manifolds backward in time. The leftmost symbols of the unstable manifold of $F_{L}$ are "A," "B," and "C." The remaining segments are labeled "B," "-," “+," and "C." The two segments of the unstable manifold of $F_{L}$ are now cut into four segments that are labeled "A," "Ā," "+," and "C."

of $F_{L}$ as "A," "B," and "C" (we follow the notation in Ref. [3]). We label the two remaining pieces of the unstable manifold of $F_{R}$ as "+" and "C." In Fig. 4(b) we show the result of two iterations of the PSS. In this second iteration, we need to introduce two additional symbols to fully determine the symbolic dynamics. The leftmost symbols of the unstable manifold of $F_{L}$ are again "A," "B," and "C" because these segments are formed in exactly the same way as in Fig. 4(a). The segment "B-C" in Fig. 4(a) is now cut into four segments, which we label "B," "-," "+," and "C." The symbols "+" and " $C$ " are formed in a manner similar to their formation in Fig. 4(a). The two segments of the unstable manifold of $F_{R}$ are now cut into four segments that are labeled "A," " $\bar{A}$," "+," and "C." The symbols "-." and "+" both branch into the symbols $A$ and $\bar{A}$. When following the phase space flow under iterations of the PSS, it proved useful to distinguish "-_" and "+" because they give mirror image results. However, when counting the number of symbols $A$ and $\bar{A}$ that emerge from each branch, the distinction between "-" and " + " is not important.

Given these symbols, we can form a branching tree of symbols that show the fractal structure that emerges with successive iterations of the PSS. The branching tree for particles incident from the left is shown in Fig. 5(a) for four iterations of the PSS. In Fig. 5(a) we retain both symbols "_" and "+." Lines with symbol $\sqrt{ }$ (red online) indicate that the particle is scattered toward $F_{L}$ (is of $S_{L}$ type), and 


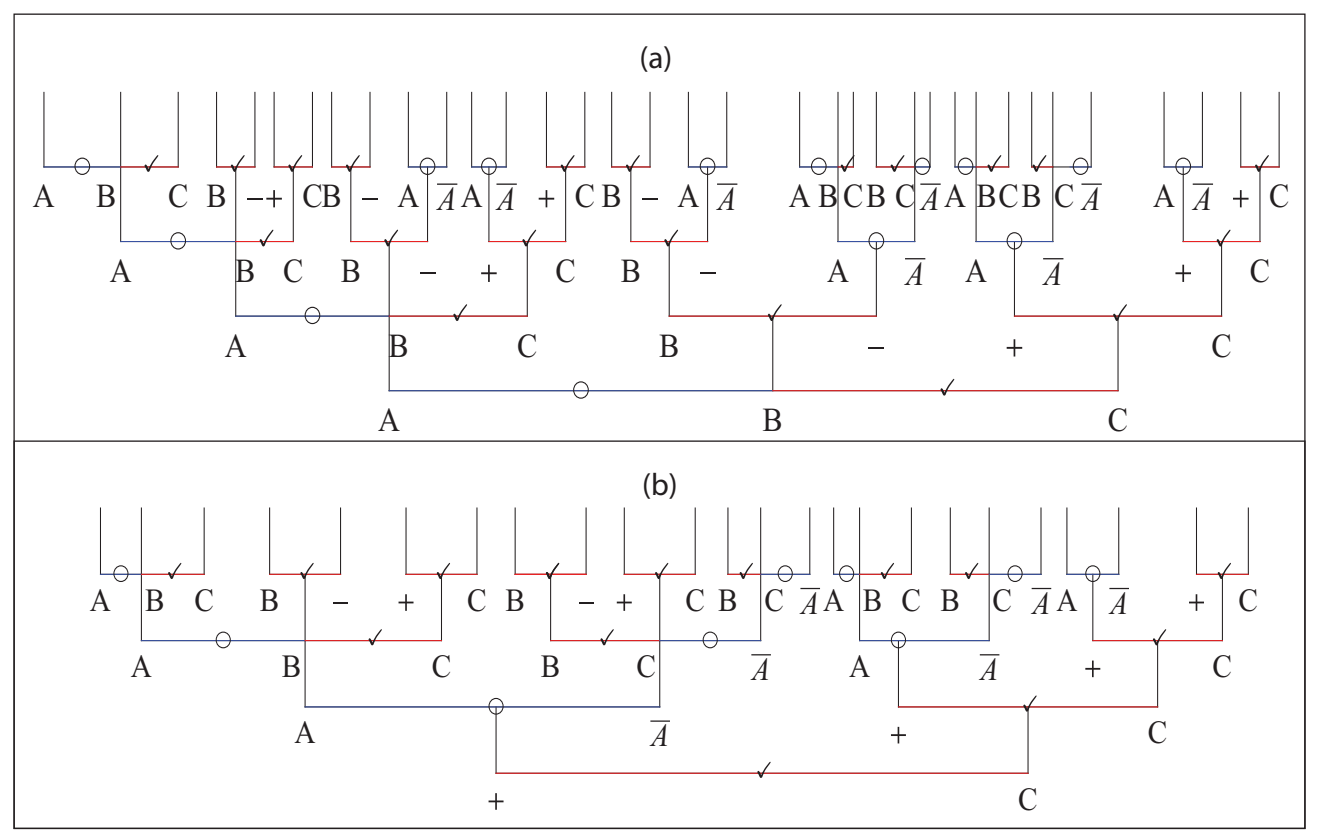

FIG. 5. (Color online) (a) The branching tree for symbolic dynamics associated with scattering from the left. (b) The branching tree for scattering from the right.

lines with symbol $\circ$ (blue online) indicates that it is scattered toward $F_{R}$ (is of $S_{R}$ type). The branching tree for particles incident from the right is shown in Fig. 5(b). We can now represent the branching trees in terms of a transfer matrix $\mathbf{T}_{6}$ that acts on a column matrix $\mathbf{s}_{6}=(A, B, C,+,-, \bar{A})^{T}(T$ denotes transpose) formed with the six symbols that make up the symbolic dynamics for this system. The transfer matrices $\mathbf{T}_{5}$ and $\mathbf{T}_{6}$ are given in Eq. (3):

$\mathbf{T}_{6}=\left(\begin{array}{llllll}1 & 0 & 0 & 1 & 1 & 0 \\ 1 & 1 & 0 & 0 & 0 & 1 \\ 1 & 0 & 1 & 0 & 0 & 1 \\ 0 & 0 & 1 & 0 & 0 & 0 \\ 0 & 1 & 0 & 0 & 0 & 0 \\ 0 & 0 & 0 & 1 & 1 & 1\end{array}\right), \mathbf{T}_{5}=\left(\begin{array}{lllll}1 & 0 & 0 & 1 & 0 \\ 1 & 1 & 0 & 0 & 1 \\ 1 & 0 & 1 & 0 & 1 \\ 0 & 1 & 1 & 0 & 0 \\ 0 & 0 & 0 & 1 & 1\end{array}\right)$

If we do not distinguish the symbols "-" and "+" and everywhere replace symbol "_" with "+," then the transfer matrix becomes a $5 \times 5$ matrix $\mathbf{T}_{5}$, which is also shown in Eq. (3). The transfer matrix $\mathbf{T}_{5}$ acts on a column matrix $\mathbf{s}_{5}=(A, B, C,+, \bar{A})^{T}$.

The transfer matrices $\mathbf{T}_{\alpha}(\alpha=5,6)$ are not self-adjoint. Therefore, $\mathbf{T}_{\alpha}$ will have $\alpha$ left eigenvectors $\boldsymbol{\psi}_{j}^{(\alpha)}, \alpha$ right eigenvectors $\boldsymbol{\phi}_{j}^{(\alpha)}$, and $\alpha$ eigenvalues $\lambda_{j}^{(\alpha)}$, where $j=$ $1, \ldots, \alpha$. The eigenvectors satisfy orthonormality conditions $\boldsymbol{\psi}_{j}^{(\alpha)} \cdot \boldsymbol{\phi}_{j^{\prime}}^{(\alpha)}=\delta_{j, j^{\prime}}$. In terms of these eigenvalues and left and right eigenvectors, the transfer matrix can be written $\mathbf{T}_{\alpha}=\sum_{j=1}^{\alpha} \lambda_{j}^{(\alpha)} \boldsymbol{\phi}_{j}^{(\alpha)} \cdot \boldsymbol{\psi}_{j}^{(\alpha)}$. For both $\alpha=5$ and 6 , there is one eigenvalue with value $\lambda_{1}^{(\alpha)}=2.3146$, while for all the other eigenvalues $\operatorname{Re}\left[\lambda_{j}^{(\alpha)}\right] \leqslant 1, j=2, \ldots, \alpha$. Therefore, when the transfer matrix acts $n$ times, we obtain $\mathbf{T}_{\alpha}^{n} \rightarrow\left(\lambda_{1}^{(\alpha)}\right)^{n} \boldsymbol{\phi}_{1}^{(\alpha)} \cdot \boldsymbol{\psi}_{1}^{(\alpha)}$ as $n \rightarrow \infty$. The left and right eigenvectors of $\mathbf{T}_{6}$ and $\mathbf{T}_{5}$ are

$$
\begin{aligned}
& \boldsymbol{\psi}_{1}^{(6)}=(0.441,0.290,0.290,0.381,0.381,0.441), \\
& \boldsymbol{\phi}_{1}^{(6)}=(0.441,0.671,0.671,0.290,0.290,0.441)^{T}
\end{aligned}
$$

and

$$
\begin{gathered}
\psi_{1}^{(5)}=(0.441,0.290,0.290,0.763,0.441), \\
\phi_{1}^{(5)}=(0.441,0.671,0.671,0.580,0.441)^{T},
\end{gathered}
$$

respectively. Note that, since the transfer matrices are known exactly, the accuracy of these numbers is limited only by the accuracy of the matrix solver.

We can now reproduce the number of each of the symbols "A," "B," "C," "+," ",$- "$ " $\bar{A}$ " at level $n$ in the branching tree for scattering from the left by allowing the transfer matrix to act $n-1$ times on the initial partition $\mathbf{s}_{1}^{L}=(1,1,1,0,0,0)^{T}$ of the unstable manifold of $F_{L}$ [see Fig. 4(a)]. We obtain $\mathbf{T}_{6}^{n-1} \cdot \mathbf{s}_{1}^{L}=$ $1.021(2.315)^{n-1} \boldsymbol{\phi}_{1}^{(6)}$. Similarly, if we allow the transfer matrix to act $n-1$ times on the initial partition $\mathbf{s}_{1}^{R}=(0,0,1,1,0,0)^{T}$ of the unstable manifold of $F_{R}$ [see Fig. 4(a)] for scattering from the right, we obtain $\mathbf{T}_{6}^{n-1} \cdot \mathbf{s}_{1}^{R}=0.671(2.315)^{n-1} \boldsymbol{\phi}_{1}^{(6)}$. (A similar analysis can be performed on $\mathbf{T}_{5}$.)

It takes only a few steps up the branching tree for scattering from the left, or from the right, for the fractional distribution of symbols "A," "B," "C," "+," " -," "A A" to be determined by the fractional distribution of symbols in the right eigenvector $\phi_{1}$. Thus, for both incident directions, the branching trees, in regard to the fraction of each type of symbol present, become the same. The fractions are $f_{A}=0.157, f_{B}=0.239, f_{C}=$ $0.239, f_{+}=0.103, f_{-}=0.103$, and $f_{\bar{A}}=0.157$.

We now can use this information, and the information in Fig. 5(b), to obtain a rough estimate of the likelihood that an incident particle, which gets caught up in the fractal 
structure, gets transmitted or reflected. First, remember that $\sqrt{ }$ (o) indicates a gap containing trajectories that ultimately travel to $-\infty$ and are of $S_{L}$ type (travel to $+\infty$ and are of $S_{R}$ type). From Fig. 5(b) we see that intervals of type "A" and " $\bar{A}$ " in an unstable manifold, in the next iteration of the map, will contain one $S_{L}$ gap and one $S_{R}$ gap. Intervals of type "B" and "C," in the next iteration of the map, will contain two $S_{L}$ gaps. Intervals of type "+" and "-," in the next iteration of the map, will contain two $S_{R}$ gaps. The fraction of $S_{L}$ gaps, in the next iteration of the map, is 0.636 , and the fraction of $S_{R}$ gaps is 0.364 . Thus, for particles incident from the left, $36.4 \%$ of the gaps get transmitted, and $63.6 \%$ of the gaps get reflected. For particles incident from the right, $63.6 \%$ of the gaps get transmitted, and $36.4 \%$ get reflected.

For a given line of initial points that cross a tendril in the asymptotic region, the percentage of gaps transmitted or reflected gives an indication of the asymmetry of the scattering process, but it does not give the actual value for the percentage of initial points transmitted or reflected. Lines of initial points can cross tendrils in the asymptotic region in different places, at different angles, etc. The stepping time plots, such as that shown in Fig. 3(c), do give that information because they distinguish the initial points being scattered to the right $\left(S_{R}\right.$ type and colored blue online) from the initial points being scattered to the left ( $S_{L}$ type and colored red online). By adding up the length of the $S_{R}$ lines and the $S_{L}$ lines in Fig. 3(b), we find that, for the line of initial conditions shown in Fig. 2, of those crossing the tendril, $44.24 \%$ are transmitted and $55.76 \%$ are reflected.

\section{v. CONCLUSIONS}

The PSS that we use (plot $p$ and $x$ each time $\phi=$ $\pi / 2+n 2 \pi$, where integer $n=0,1,2, \ldots)$ to analyze the scattering dynamics corresponds to a particular phase of the radiation field at time $t=0$ when we begin to follow our trajectories. If the phase of the field is different at time $t=0$, then the asymmetric scattering properties will differ in detail but will still be asymmetric. The procedure described here can be applied to any choice of initial phase of the radiation field. The symbolic dynamics of the scattering process, and the resulting transfer matrix, can provide an important tool for disentangling reflection from transmission in such scattering processes.

\section{ACKNOWLEDGMENT}

The authors wish to thank the Robert A. Welch Foundation (Grant No. F-1051) for support of this work.
[1] C. Jung and H. J. Scholz, J. Phys. A 20, 3607 (1987); 21, 2301 (1988).

[2] B. Rückerl and C. Jung, J. Phys. A 27, 55 (1994).

[3] A. Emmanouilidou, C. Jung, and L. E. Reichl, Phys. Rev. E 68, 046207 (2003).

[4] C. Jung and A. Emmanouilidou, Chaos 15, 023101 (2005).

[5] A. Emmanouilidou and C. Jung, Phys. Rev. E 73, 016219 (2006).

[6] A. M. Barr, K. Na, L. E. Reichl, and C. Jung, Phys. Rev. E 79, 026215 (2009).

[7] B. Eckhardt, Physica D 33, 89 (1988).

[8] C. Jung and T. H. Seligman, Phys. Rep. 285, 77 (1997).
[9] A. Emmanouilidou and L. E. Reichl, Phys. Rev. A 65, 033405 (2002).

[10] K. Na, D. Jarukanont, and L. E. Reichl, Phys. Rev. E 77, 046208 (2008).

[11] H. A. Kramers, Collected Scientific Papers (North-Holland, Amsterdam, 1956), p. 272.

[12] W. C. Henneberger, Phys. Rev. Lett. 21, 838 (1968).

[13] H. Goldstein, Classical Mechanics, 2nd ed. (Addison-Wesley, Reading MA, 1980).

[14] G. Yao and S.-I. Chu, Phys. Rev. A 45, 6735 (1992).

[15] M. Marinescu and M. Gavrila, Phys. Rev. A 53, 2513 (1996).

[16] A. S. Fearnside, R. M. Potvliege, and R. Shakeshaft, Phys. Rev. A 51, 1471 (1995). 